\title{
Locally advanced gastroesophageal junction cancer with pathological complete response to neoadjuvant therapy: a case report and literature review
}

\author{
Xiaoying Li", Qian Huang", Yanna Lei, Xiufeng Zheng, Shuang Dai, Weibing Leng, Ming Liu \\ Department of Abdominal Oncology, West China Hospital, Sichuan University, Chengdu, China \\ \#These authors contributed equally to this work. \\ Correspondence to: Ming Liu, PhD, Professor, Department of Abdominal Oncology, West China Hospital, Sichuan University, Chengdu 610041, \\ China. Email: mingliu721@aliyun.com.
}

\begin{abstract}
Most gastric cancer and gastroesophageal junction carcinoma (GEJ) patients are already in the advanced stage at the time of diagnosis. Thus, the probability of radical gastrectomy is low, and surgical treatment alone has a poor prognosis due to the high recurrence rate. In order to reduce the recurrence and distant metastasis after surgery, there have been many attempts made to improve the perioperative treatment of advanced localized gastric cancer, but no uniform criteria exist. Over recent years, immunotherapy has revolutionized cancer treatment, and immune checkpoint inhibitors (ICIs) have shown excellent efficacy across various types of tumors, becoming a potential treatment after surgery, chemotherapy, radiotherapy, and targeted therapy. However, the efficacy of single-agent ICIs for gastric cancer is still unsatisfactory. As comprehensive, chemotherapy-based treatment has become the standard care for locally advanced gastric cancer, exploring combination treatment with immune checkpoint inhibitors (ICIs) may be valuable to improving survival outcomes. Here, we report a 66-year-old male with dysphagia diagnosed with GEJ and was defined as clinical stage (cT4N2M0) and Siewert type II, characterized as mismatch repair proficient (pMMR) and programmed cell death ligand-1 (PD-L1) negative; surprisingly, with anti-PD-1 antibody plus SOX (S-1: a combination of tegafur, gimeracil, and oteracil+ oxaliplatin) as perioperative therapy, the patient achieved pathological complete remission (pCR), which indicates that the addition of ICIs to chemotherapy as a perioperative comprehensive treatment might provide a promising strategy option for GEJ. In addition, we review the current status of perioperative comprehensive treatment, in hope that this may provide some reference value for clinical decision-making.
\end{abstract}

Keywords: Case report; gastric and gastroesophageal junction cancer; PD-1; neoadjuvant therapy

Submitted Dec 18, 2020. Accepted for publication Mar 23, 2021.

doi: 10.21037/atm-21-434

View this article at: http://dx.doi.org/10.21037/atm-21-434

\section{Introduction}

Gastric cancer remains a major cause of cancer-related mortality and incidence, with more than 1 million new cases and almost 800,000 deaths occurring in 2018, not only making it the fifth most common cancer, but also the third leading source of cancer-related mortality worldwide (1). In China, according to a retrospective study of 2015 , the age-standardized 5 -year relative survival for all cancers was $30.9 \%$, while that of gastric cancer was $27.4 \%$ (2). As gastric cancer is rarely detected early, the morbidity and mortality rates are significantly high, with the 5 -year survival rate of most countries fluctuating between $20 \%$ and $40 \%(3,4)$. The treatment modalities for locally advanced gastric cancer and gastroesophageal junction (GEJ) carcinoma vary regionally. Europe emphasizes perioperative chemotherapy, North America advocates postoperative synchronization chemoradiotherapy, while Asia prefers D2 radical surgery combined with postoperative adjuvant chemotherapy, 
with surgery remaining a curative treatment in the world. A variety of treatment methods, such as neoadjuvant, adjuvant, and perioperative regimens, including different chemotherapy and radiotherapy combinations, are used as multimodality treatments to reduce local and distant recurrence after gastrectomy and to improve survival rates. However, the optimal systemic strategy for gastric cancer/ GEJ has not been standardized. There were different treatment modalities of neoadjuvant combination therapy, such as combined thymalin, targeted drugs or hyperthermic intraperitoneal perfusion chemotherapy (5-10). According to previous clinical studies conducted on advanced gastric patients, only a small percentage of patients with currently recognized immunotherapy sensitive biological markers such as PD-L1 (11), tumor mutation burden (TMB) $(12,13)$ and MSI-H/dMMR show a good response to immune checkpoint inhibitors (ICIs) $(14,15)$, and accurate prediction markers of efficacy are still unsatisfactory. Therefore, combination of perioperative ICIs and chemotherapy could be a novel therapy to increase response rate and resectability and reduce recurrence rate in patients with locally advanced gastric cancer/GEJ, and there were planned clinical trials to test efficacy and safety of perioperative chemotherapy plus PD-1 antibody in gastric cancer like (NCT04367025).

According to previous studies, mismatch repair proficient (pMMR) is less sensitive to immunotherapy because it lacks sufficient neoantigens compared to mismatch repair deficiency (dMMR) $(14,15)$. In this case, we conducted a retrospective analysis of the characteristics of a $\mathrm{pMMR}, \mathrm{PD}-$ L1-negative, Epstein-Barr virus (EBV)-negative patient, who was supposed to be relatively insensitive to immunotherapy and found he still have a good response to anti-PD-1 antibody combined with chemotherapy, indicating other mechanisms than MSI, PD-L1 positive or EBV infection may account for the responsiveness of gastric cancer/GEJ to ICIs. To the best of our knowledge, this is the first case report of pathological complete remission of locally advanced GEJ cancer patient after ICIs combined with neoadjuvant chemotherapy in perioperative setting, with characteristics of pMMR, PD-L1-negative, EBV-negative. Chemotherapy may change the immune environment of primary tumor to induce immune activation thus rendering immunotherapy therapeutic. We also briefly review the current status of the perioperative treatment modalities in gastric cancer, in order to provide some reference guidance in clinical decisionmaking. We present the following article in accordance with the CARE reporting checklist (available at http://dx.doi. org/10.21037/atm-21-434).

\section{Case presentation}

A 66-year-old male presented with dysphagia without obvious inducement for more than 1 year (September 2019), which was progressively aggravated and accompanied by lower esophageal infarction. The patient has no family genetic history or other special medical history. Contrastenhanced computed tomography (CT) of the abdomen was performed: the wall of the lower esophagus and cardia was thickened and enhanced, and multiple lymph nodes beside the lower esophagus, hepatogastric ligament, and para-aortic region were found, with some enlargement (Figure 1A,B,C). He underwent gastroscopy, and a cardiac mass was found (Figure 1D), with pathological biopsy indicating adenocarcinoma. Based on the above findings, the adenocarcinoma of gastric was defined as clinical stage (cT4N2M0) and Siewert type II, which was considered resectable after neoadjuvant chemotherapy, according to American Joint Committee on Cancer (AJCC)/Union for International Cancer Control (UICC) 8th Tumor Node Metastasis (TNM) staging classification. Many clinic trials have been carried out for immunotherapy in the fields of firstline treatment of advanced gastric cancer/GEJ and revealed encouraging results, the objective response rate (ORR) in the cohort 2 study of KEYNOTE059 was $60.0 \%$ and ORR in ATTRACTION-04 was $67 \%$ when ICIs combined with chemotherapy used as first-line therapy (16-18). Camrelizumab (anti-PD-1 antibody, SHR-1210) showed encouraging efficacy in Chinese patients with recurrent or metastatic gastric cancer/GEJ cancer, who were refractory or intolerant to previous chemotherapy (ORR was 23.3\% regardless of the PD-L1 status), including mismatch repair subgroup (ORR was 30\%) (19). Because the high efficiency and low toxicity of ICIs have been confirmed by previous clinical studies and with the respect to patient's wishes, camrelizumab was applied into perioperative therapy without molecular biology test. No treatment was taken before neoadjuvant therapy. Rule out the taboo of chemotherapy, from November 2019 to January 2020, the patient received 3 cycles of chemotherapy with oxaliplatin $130 \mathrm{mg} / \mathrm{m}^{2}$ on day 1 and $50 \mathrm{mg} \mathrm{S}-1$ (a combination of tegafur, gimeracil, and oteracil) twice a day from days 1-14 in each 3-week period, which is called SOX regimen, meanwhile plus camrelizumab $200 \mathrm{mg}$ ivgtt day1, q3w. The treatment was tolerated well, no immune-related adverse effect was observed based on examination of blood toxicity and multiple organ functions. After three cycles of treatment, a whole abdominal CT scan (Figure 1E,F,G) taken on March 25, 2020, showed that the 

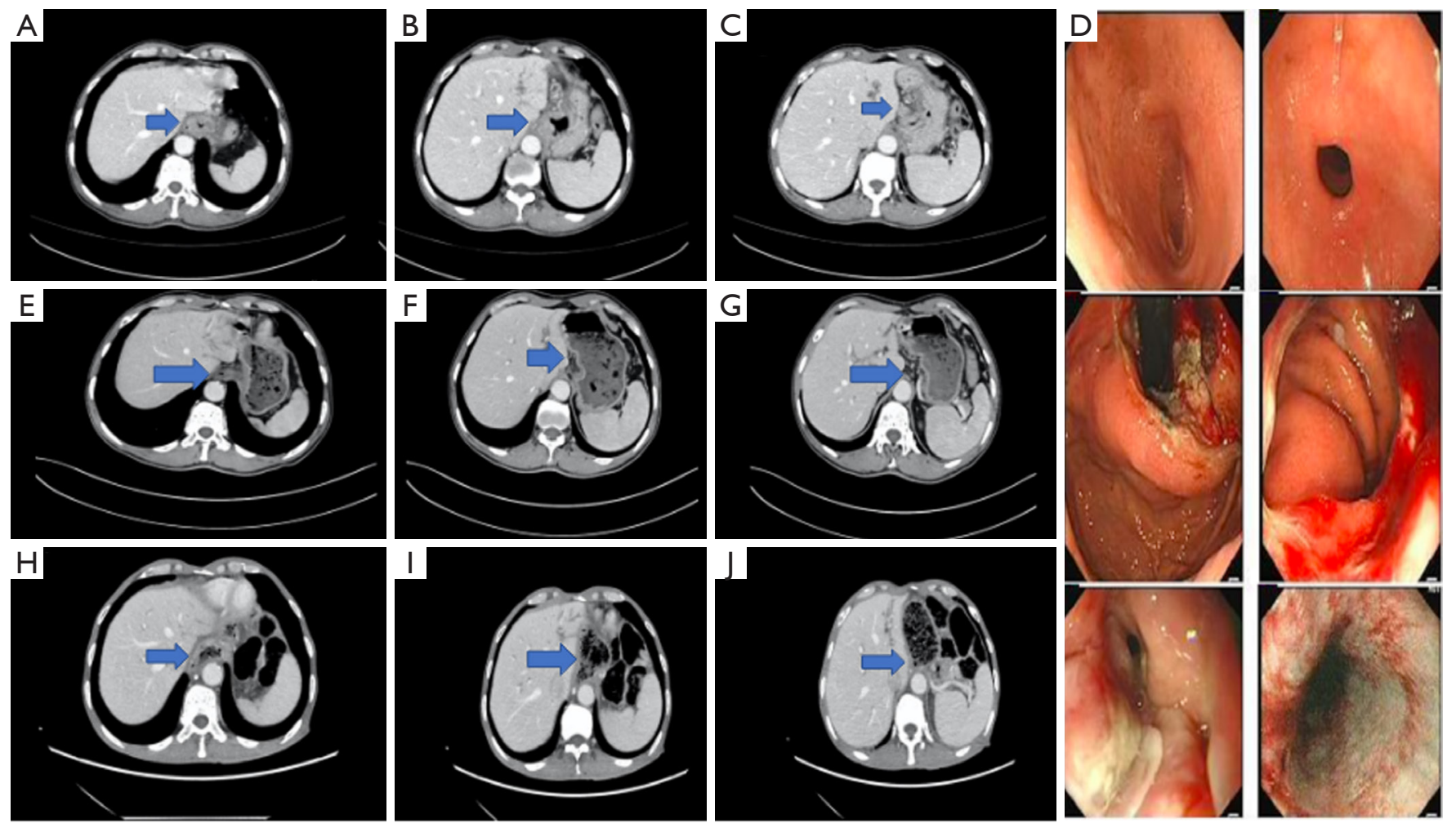

Figure 1 Computed tomography scan of the tumors and regional lymph nodes (blue arrows) before and after anti-PD-1 therapy combined with chemotherapy as well as after surgery. Computed tomography images of primary lesions and regional lymph nodes before (A,B,C) and after (E,F,G) combined immunotherapy and chemotherapy, and after surgery (H,I,J). Primary tumor and metastatic lymph nodes are no longer visible after immune-chemotherapy. Gastroscopy revealed a mass adjacent to the cardia before neoadjuvant therapy (D).

wall of the lower esophagus and cardia was not thickened, and that the small lymph nodes in the hepatogastric ligament were not enlarged. The efficacy of treatment was defined as clinical partial response (PR). On April 3, 2020, the patient underwent radical gastrectomy with D2 lymphadenectomy, and postoperative pathological examination indicated a pathological staging of ypTONOM0 and pathological complete response (pCR). Repeated CT examination after surgery did not show tumor recurrence or metastasis (Figure $1 \mathrm{H}, I, \mathcal{F}$ ). We used immunohistochemistry (IHC) to explore the relationship between the immune microenvironment and the curative effect of anti-PD-1 therapy in gastroscopic biopsy specimens. We used IHC to assess the expression of mismatch repair proteins, this patient was revealed to have pMMR status (Figure 2) and was mismatch repair proficient, MLH1 positive, MSH2 positive, PMS2 positive, and MSH6 positive; the patients was also human epidermal growth factor receptor 2 (HER2) negative, and the positive rate of
Ki67 was about $60 \%$. IHC assay of tumor biopsy samples revealed PD-L1-negative status (PD-L1 IHC 22C3, 1:100, Dako) (Figure 3A), and EBV-encode small RNA in situ hybridization indicated an EBV-negative status (Figure 3B). Hematoxylin and eosin (HE) staining of tumor specimens before surgery suggested that adenocarcinoma (Figure 4A). Postoperative pathology showed no residual tumor cells, and a pathological complete response was achieved (Figure 4B), so we also conducted IHC assays of CD3( clone LN10, 1:100), CD45RO(clone UCHL1, 1:50), CD8 (clone EP334, 1:200) and FOXP3(clone 236A/E 1:100) on preoperative biopsy specimens to explore the tumorinfiltrating lymphocytes (TILS) distribution, and found a large number of $\mathrm{CD} 3+\mathrm{T}$ lymphocytes at the edge of the tumor (Figure 4C), CD8+ T lymphocytes was minimally expressed (Figure 4D), with CD45RO+ T lymphocytes also being visible in tumor specimens (Figure 4E), while TILs were negative for FOXP3 (Figure $4 F$ ). Since there was no pathological specimen, the tumor mutation burden (TMB) 


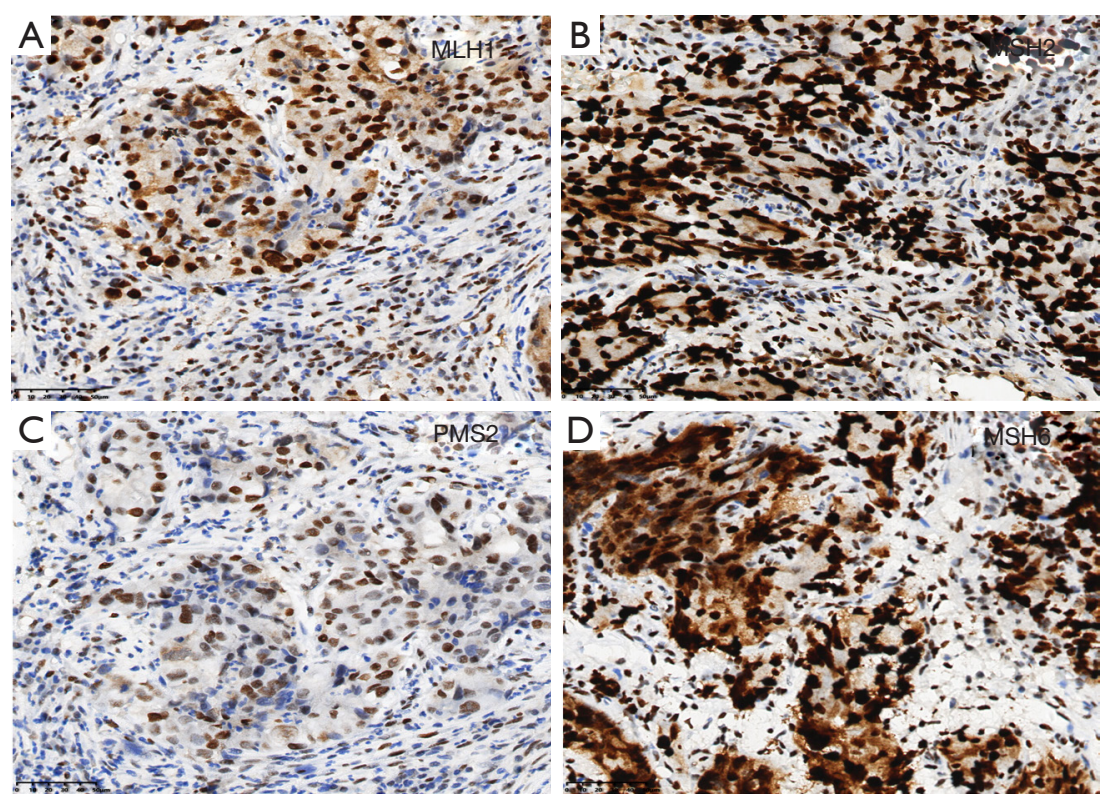

Figure 2 Immunohistochemistry of mismatch repair proteins. The tumor cells are positive for MLH1 (A), MSH2 (B), PMS2 (C), and MSH6 (D). Scale bar: $50 \mu \mathrm{m}$ Original magnification: 400×.
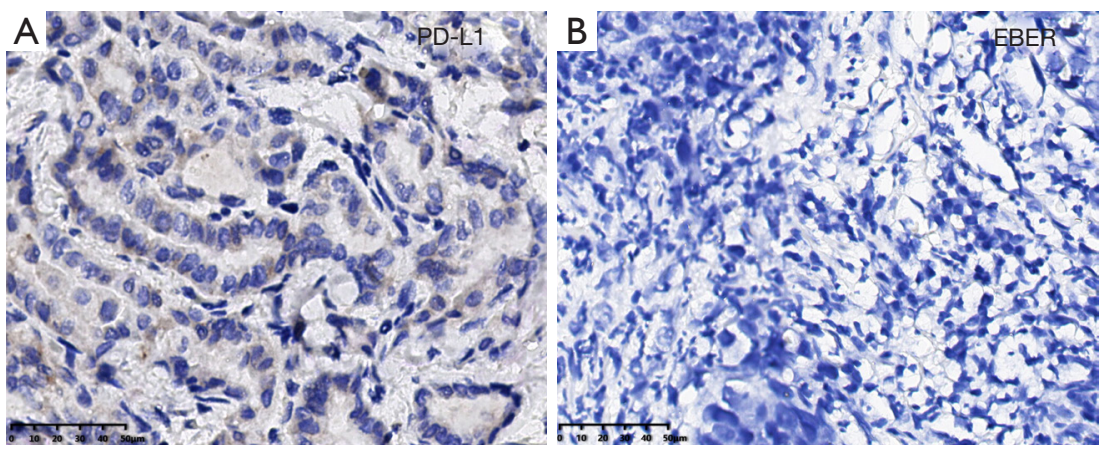

Figure 3 Immunohistochemistry assay of PD-L1 protein and EBV status. PD-L1 (B7-H1) was negative in tumor tissue by immunohistochemistry assay (A) and EBV virus was absent by using EBV-encode small RNA (EBERs) in situ hybridization (B). Scale bar: $50 \mu \mathrm{m}$. Original magnification: $400 \times$.

was not assessed in the patient. As the patient insisted on continuing postoperative treatment, and the compliance and tolerance of the patient are very high. He received 3 cycles of postoperative chemotherapy plus camrelizumab. Thus far, no obvious signs of recurrence have been observed during the regular follow-up of this patient (Figure 5). All procedures performed in studies involving human participants were in accordance with the ethical standards of the institutional and/or national research committee(s) and with the Helsinki Declaration (as revised in 2013). Written informed consent was obtained from the patient for publication of this case report and any accompanying images.

\section{Literature review}

\section{The role of neoadjuvant therapy and immunotherapy in the perioperative setting}

Neoadjuvant chemotherapy, also known as preoperative chemotherapy, appears to associated with a greater possibility of complete resection and a more favorable post-treatment tumor stage. It also has been shown to increase survival 

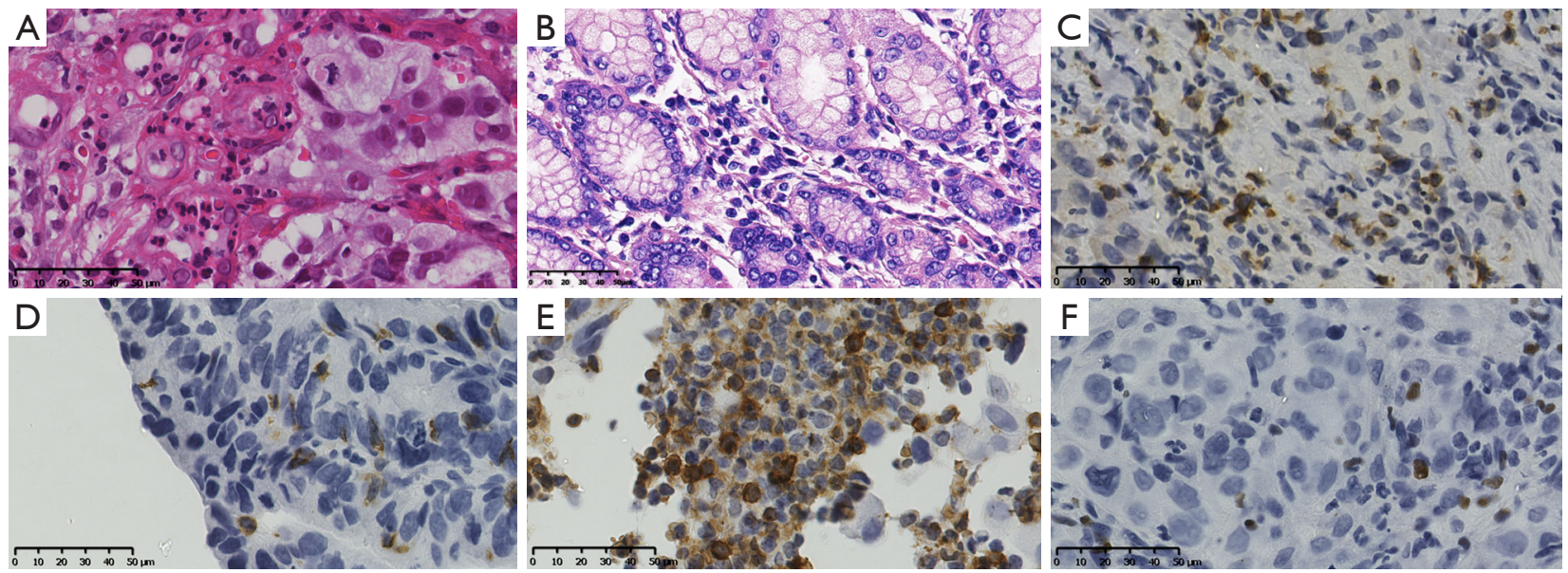

Figure 4 The histopathology of the primary tumor specimens and the immunohistochemistry of the primary tumor biopsy specimens as well as postoperative pathology. Hematoxylin and eosin (HE) staining of tumor specimens before surgery (A). Postoperative pathology showed no residual tumor cells, and a pathological complete response was achieved (B). CD3+ tumor-infiltrating lymphocytes were observed, with a tumor proportion score (TPS) of around 20\%. (C) CD8+ tumor-infiltrating lymphocytes had a TPS of about 1\% (D). The CD45RO+ tumor-infiltrating lymphocytes TPS score was about 15\% (E). FOXP3 tumor-infiltrating lymphocytes was not observed (F). Scale bar: $50 \mu \mathrm{m}$. Magnification: 400×.

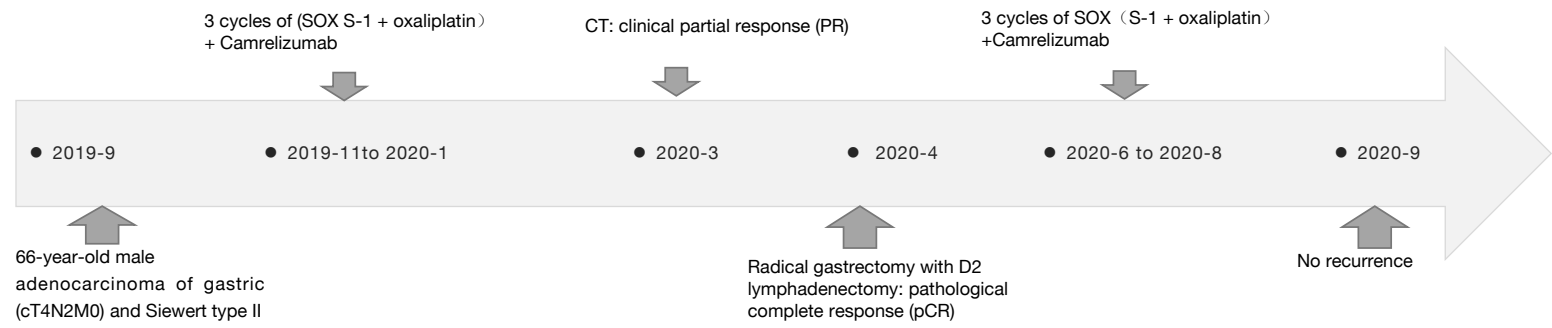

Figure 5 Timeline of diagnosis and treatment.

compared to surgery alone, but not when perioperative complications occur (20). In the European landmark phase III MAGIC trial (21), it was found that, as compared with the surgery group, the perioperative chemotherapy group [epirubicin, cisplatin, and infused fluorouracil (ECF)] showed a better overall survival (OS) and a greater progressionfree survival (PFS), which established the perioperative ECF chemotherapy regimen as the standard of treatment in resectable GC and GEJ patients. Later, an anthracyclinefree regimen phase III trial (FNCLCC/FFCD ACCORD) was conducted to evaluate the benefit of perioperative fluorouracil plus cisplatin (CS) in OS among patients with resectable gastroesophageal adenocarcinoma. It found that, when compared with the surgery group (22), the CS group had a better 5 -year OS rate, and a better 5 -year diseasefree survival (DFS). Given the efficacy of CS, other two- drug regimens, including S-1 plus oxaliplatin, capecitabine plus oxaliplatin, or the FOLFOX regimen (fluorouracil, leucovorin, and oxaliplatin) were also confirmed to benefit patients in the adjuvant setting (23-25), and have thus been applied in neoadjuvant therapy as well. A more recent study found that OS was increased in patients treated with a perioperative FLOT (26) (fluorouracil plus leucovorin, oxaliplatin and docetaxel) regimen, when compared with patients treated with the perioperative ECF/ECX (epirubicin, cisplatin, and capecitabine) regimen. Furthermore, median OS was improved from 35 to 50 months, demonstrating the superiority of the FLOT protocol and its ability to not only improve histopathological tumor response but also increase OS. The MAGIC (UK), FNCLCC/FFCD ACCORD (France), and FLOT-4 (German) trials established neoadjuvant chemotherapy as the standard mode of therapy 
in Europe. However, there is still lack of good phase III clinical studies in the study of neoadjuvant chemotherapy, and most of the research regimens are replacements for chemotherapeutic drugs, deriving from chemotherapy for advanced gastric cancer; therefore, researches into the selection of the best neoadjuvant chemotherapy drugs and regimens remains a critical need for patients with gastric cancer. According to The European Society for Medical Oncology (ESMO) clinical guideline, a perioperative strategy is recommended for most Western patients who are diagnosed as T3-4 or who have local lymph node metastasis (27), however, there is current evidence which suggests that not all patients with cT2N0M0 should undergo such treatment, but only those with a high risk factors for recurrence $(28,29)$.

The success of neoadjuvant chemotherapy has encouraged clinical radiologists. In recent years, many scholars have begun to deeply explore the benefits of radiotherapy in the neoadjuvant setting in locally advanced gastric cancer patients. Chemoradiotherapy was evaluated in two large-scale clinical trials (CALGB 9781 and CROSS) $(30,31)$, and results indicated that preoperative chemoradiotherapy improved survival and was well tolerated, thus supporting the application of chemoradiotherapy for esophageal adenocarcinoma and GEJ tumor in the neoadjuvant setting. When we can expect radiotherapy to add to the efficacy of preoperative or postoperative chemotherapy-if we can expect it at all-is not clear, and this strategy is currently not recommended in gastric cancer. However, relevant research on patients with insufficient D1+ or D2 lymphadenectomy, or even incomplete resection, found that postoperative chemoradiotherapy could undoubtedly improve the OS rates $(32,33)$. Several randomized control trials comparing neoadjuvant chemotherapy to preoperative chemoradiotherapy are currently ongoing, with the chemotherapy regimens scrutinize mainly being ECF, FLOT, or the DOC (docetaxel, capecitabine, oxaliplatin) three-drug regimen (NCT02509286, NCT02931890, NCT03013010, NCT04375605) (34,35). It is hoped that these studies can identify the most optimal treatment regimen in patients with resectable gastric adenocarcinoma.

A number of phase II clinical studies have assessed the efficacies of different regimens of combined chemotherapy and immunotherapy in the perioperative setting of gastric cancer and GEJ. One clinical trial (NCT04006262) is presently recruiting and aims to assess the perioperative role of immune checkpoint inhibitors (ICIs) in mismatch repair deficiency (dMMR) and/or microsatellite instability (MSI) patients (36). This may provide more guidance for the management of localized MSI/dMMR in gastric cancer/GEJ patients. Meanwhile, the EORTC VESTIGE (NCT03443856) will be conducted in high risk patients undergoing neoadjuvant chemotherapy and surgery, and is aimed at improving DFS by combining ICIs (nivolumab and low dose ipilimumab) with chemotherapy in the adjuvant setting (37). Additionally, the IMAGINE trial (NCT04062656) is a four-arm, chemotherapy controlled modular trial, and its aim is to evaluate perioperative chemoimmunotherapy $v s$. immunotherapy $v s$. chemotherapy in gastric cancer and esophagogastric junction cancer patients. Another phase II trial (NCT03878472) will be performed with locally advanced proximal gastric cancer patients and aims to evaluate the benefit of anti-PD-1 in combination with apatinib [a novel anti-angiogenesis vascular endothelial growth factor 2 (VEGFR2) tyrosine kinase inhibitor] with or without chemotherapy (S1/oxaliplatin) in the preoperative setting. Other ICIs, such as avelumab (an anti-PD-L1 monoclonal antibody), atezolizumab (an antiCTLA-4 monoclonal antibody) will also be explored (NCT03399071, NCT03448835, NCT03421288). Of these atezolizumab trials, one (NCT03421288) is assessing atezolizumab combined with FLOT in perioperative treatment of patients with gastric adenocarcinoma (38).

\section{Current status of immunotherapy in gastric cancer}

Immunotherapy has changed the landscape of cancer treatment, with ICIs having shown promising clinical benefit in melanoma (39-42). Outcomes from clinical studies, such as the KEYNOTE-012, ATTRACTION-2 and KEYNOTE-059 trials, support the efficacy of ICIs in a subset of patients with metastatic gastric cancer $(16,43,44)$. Consequently, pembrolizumab has been given US Food and Drug Administration (FDA) approval for the treatment of gastric cancer and GEJ adenocarcinoma patients in the recurrent, locally advanced or metastatic setting. Although pembrolizumab did not surpass paclitaxel in second-line therapy in the KEYNOTE-061trail (45), and pembrolizumab in combination with chemotherapy (FP, cisplatin and fluorouracil) did not prolong the OS or PFS in first-line treatment for advanced gastric cancer/GEJ with a PD-L1 combined positivity score CPS of 1 (46). Another anti-PD-1 (nivolumab) was applied in clinic according to the ATTRACTION-04 and CheckMate-649 trials. In view of the low effective rate of mono-immunotherapy 
for gastric cancer, immunotherapy combined with chemotherapy or targeted therapy might be more beneficial than monotherapy, with some clinical trials attesting to this efficacy. For example, the ATTRACTION-4 trial showed that nivolumab in combination with chemotherapy has a tolerable safety profile and reported encouraging results, with objective response rates (ORRs) of $57.1 \%$ and $76.5 \%$ and a median PFS of 9.7 and 10.6 months with SOX (S-1, oxaliplatin) and CAPOX (capecitabine, oxaliplatin), respectively (17). Double immunotherapy has also demonstrated good results. For instance, in the CheckMate-032 trial (47), nivolumab plus ipilimumab exhibited clinically meaningful antitumor effects in patients with chemotherapy-refractory esophagogastric cancer, and more phase III studies evaluating this combination in earlier lines of therapy for esophagogastric cancers are underway. Immunotherapy combined with chemotherapy and targeted therapy have also shown good clinical therapeutic effects. A phase 2 study (EPOC1706) assessed the antitumor activity and safety of lenvatinib plus pembrolizumab in patients with advanced gastric cancer in the first-line or second-line settings: objective response efficacy was found in 20 of 29 patients, indicating a superior efficacy than previous single drugs and an acceptable safety profile (48). KEYNOTE811 is an ongoing phase III clinical trial (NCT03615326) comparing pembrolizumab versus placebo in the setting of trastuzumab in combination with chemotherapy (49). In addition, a study examining ramucirumab combined with pembrolizumab in patients with previously treated advanced gastric cancer or GEJ, with the primary endpoints of safety and tolerability found this regimen could offer good tolerability and potential efficacy (disease control rate, 68\%; median PFS, 5.3 months) (50). Furthermore, the phase III CheckMate-649 clinical study, the largest clinical study conducted to date in the field of gastric and esophageal cancer, demonstrated OS and PFS benefit in a combined nivolumab and chemotherapy group for first-line treatment. The combination of nivolumab and chemotherapy will hopefully change the first-line treatment landscape for gastric/GEJ and become a new standard of care for these patients. Radioimmunotherapy is also currently being investigated, with radiotherapy having been shown to modulate the immune microenvironment of the tumor and upregulate the expression of PD-L1 on tumor and immune cells to treat patients with cancer (51). In a case report of one patient, radiotherapy combined with pembrolizumab was able to provide a disease control condition (with no further therapy) for a period of 12 months before relapse
(52), implying that radioimmunotherapy may have potential in combination therapy. Various combined treatment methods for advanced recurrent or metastatic gastric cancer are being actively explored, and positive results may provide options for the perioperative period. Further evaluation of ICIs and their combination treatments in the perioperative setting might be beneficial for patients with locally resectable disease.

\section{Discussion}

Perioperative treatment has been shown to improve survival in both esophageal and gastric trials, but the therapy regimen has not been standardized, with and Eastern and Western guidelines having disparate recommendations. Pathological regression grading is currently the most accurate way to determine the effectiveness of neoadjuvant therapy. Several clinical studies of previous neoadjuvant chemotherapies have reported $\mathrm{pCR}$ rates ranging between $6 \%$ and $20 \%$ (53-55). According to previous research, patients who achieved pCR status had significantly longer OS and recurrence-free survival (RFS) than those who did not. pCR is not only an effective indicator of good shortterm efficacy of neoadjuvant therapy, but can also better predict the recurrence, metastasis, and survival of gastric cancer patients after neoadjuvant therapy (56). Indeed, in our case report, the patient achieved pCR status after combination treatment, and thus immunotherapy may play an important role in prolonging OS. However, the gastric cancer features in heterogeneous tumor microenvironments are inconsistent in response to ICIs, so biomarkers related to treatment response need to be selected in order to guide the rational selection of appropriate patients for immunotherapy in clinical practice. The Cancer Genome Atlas Project (TCGA) categorized gastric cancer into chromosomally unstable (CIN, 50\%), microsatellite unstable (MSI, 22\%), genomically stable (GS, 20\%), and EBV (EBV, 9\%) subtypes according to molecular features, which may lay the foundation for targeted therapy trials and stratification of patients (57). Given the very low response rates to ICIs in gastric cancer patients $(16,43,45,46)$, molecular typing may provide better guidance for precision treatment. Some studies have demonstrated that microsatellite instability high (MSI-H), dMMR, programmed cell death ligand-1 (PD-L1) positive, and EBV positive are biomarkers for selecting those gastric cancer patients who may benefit from ICI application $(16,43,46,58)$.

As chemotherapy and radiation therapy has been 
shown to modulate the immune environment of the tumor microenvironment and to induce up-regulation of PD-L1 on tumors and immune cells $(51,59-62)$, there is increasing interest in the study of perioperative immune checkpoint suppression in gastric cancer and GEJ. The combination of ICIs with standard perioperative therapy is supported by previous observations on other solid tumors, which indicating ICIs can be used in combination with chemotherapy, targeted drugs, radiation therapy and other immunotherapies. With the development of ICIs, there are growing treatment options for cancer patients, but the best treatment is still unknown, thus many prospective clinical investigations are being evaluated. Early use of antiPD-1 can reduce the mortality of cancer patients, and once it works, patients may survive for a long time, or even be "cured" clinically. It has been demonstrated patients who use immunotherapy as first-line therapy and then receive other drugs have a lower risk of death than those who receive immunotherapy after other drugs (HR: 0.69) (63), meanwhile ICIs shows better safety profile with its low toxicity than chemotherapy. Therefore, its application in the early stage of cancer is worth trying.

Tumor with DNA mismatch repair (dMMR) defects are highly microsatellite unstable (MSI-H) and carry hundreds of somatic mutations that encode potential new antigens, therefore, this kind of tumor is likely to have immunogenicity and trigger the up-regulation of immune checkpoint protein. Some studies indicated that the response of ICIs correlated with TMB, dMMR status, EBV status, and programmed cell death ligand 1 (PD-L1) expression $(16,43,46,58,64)$. As gastric cancer and GEJ are potential target for ICIs, ICIs have a better safety profile than chemotherapy. The treatment algorithm of gastric cancer and GEJ has recently been enriched with the clinical application of ICIs. If the relevant characteristic biomarkers can be detected before operation and immunotherapy can be used according to the biomarkers, it may bring greater clinical benefits to the patients.

In our case, immunohistochemistry showed the following: pMMR, PMS2 positive, MSH6 positive, MLH1 positive, MSH2 positive, and HER2 negative. The Ki67positive rate was about $60 \%$. In situ hybridization indicated an EBV-negative status in the tumor cells and a PD-L1negative status, with a tumor proportion score (TPS) of $<1 \%$. However, this patient demonstrated a dramatic response to a treatment of immunotherapy combined chemotherapy and achieved pCR. In addition, neoadjuvant chemoradiotherapy was recommended since as he was
Siewert type II (65). Considering his physical tolerance, radiation was not performed and chemotherapy combined with immunotherapy was adopted. After three cycles of combination therapy, the clinical efficacy of the patient was assessed as PR, and then surgery was performed, with combination therapy continuing after surgery. More studies are needed to confirm the different combination therapy approaches, and to identify predictive and prognostic biomarkers for selecting advantaged patients with advanced gastric cancer/GEJC adenocarcinoma. In breast cancer, increased TIL concentration is thought to be associated with a survival benefit and to be able to predict response to neoadjuvant chemotherapy (66); however, its function in gastric cancer is not clear. CD45RO+ $\mathrm{T}$ lymphocytes that play secondary immune killing in vivo have auxiliary induction; the higher the degree of CD45RO infiltration, the more the number of active $\mathrm{T}$ cells, the better the prognosis. Besides CD45RO+ effector memory $\mathrm{T}$ cell phenotype seems to more abundant in patients who benefit from immunotherapy compared with non-responders (67). According to previous founding, neutrophil count level significantly increased in patients with hyperprogressive disease (HPD) (68). Neutrophils and macrophages are also found infiltrating many types of tumors, but tumorassociated neutrophils (TANs), its role is still controversial and may promote or inhibit the development of tumors (69-71). Tumor-associated macrophages (TAMs) are a major constituent of the tumor microenvironment, where they either promote the formation of an immunosuppressive microenvironment or inhibit tumorigenesis and metastasis depending direct or indirect suppression of cytotoxic $\mathrm{T}$ cell activity, generally represent a poor prognostic factor and play a complex role in GC /GEJ $(72,73)$. As it is a retrospective exploration of factors in the microenvironment that play a positive role in the effect of immunotherapy, TANs and TAMs were not examined in this study. Aiming at investigating the prognostic impact of TILs in gastric cancer, we performed IHC to evaluate the tumor microenvironment. As patients with pre-existing $\mathrm{T}$ cell infiltration into the tumor microenvironment are more likely to respond to immune checkpoint inhibition, ICIs may exert an inhibitory effect on tumor growth, and preexisting CD8+ T lymphocytes are required (74). In this patient, the $\mathrm{CD} 3+\mathrm{T}$ cells, CD8+ $\mathrm{T}$ cells, $\mathrm{C} 45 \mathrm{RO}+\mathrm{T}$ cells had TPS scores of $20 \%, 1 \%$, and $15 \%$ respectively, which might have been responsible for the dramatic response to pCR. In another study that aimed to identify tumor-immune cell association across gastroesophageal adenocarcinomas 
(GEA) subclasses, gastroesophageal adenocarcinomas exhibited a diversity of immune phenotypes (75). It has also been reported that the antigen presentation process may be a potential strategy to enhance immune activity and recognition against so-called immune desert tumors (76). ICIs are unlikely to be effective as monotherapy against intestinal and diffuse tumors lacking substantial PD-L1 expression or prominent T-cell infiltration (77), however, our patient was neither MSI nor EBV positive, and the CD8+ T cell infiltration was insufficient. According to previous studies, chemotherapy leads to the death of tumor cells, which may increase antigen presentation $(62,78)$, while other research has found that neoadjuvant chemotherapy (NACT) also induces upregulated levels of CD8+, PD1, and PD-L1 $(79,80)$. In addition, CD45RO-positive T cells seem to express more interferon- $\gamma(\mathrm{IFN}-\gamma)$, and IFN- $\gamma$ are likely to induce local immune activation (81) and the absence of immunosuppressive elements like FOXP3 $+\mathrm{T}$ cells (82). In our case, neoadjuvant chemotherapy may alter the tumor microenvironment, so that the patient could benefit from immunotherapy and achieved pCR. To date, the evidence on how the immune microenvironment affects immunotherapy response is still not clear. Thus, we do not know whether this patient achieved pCR simply as a result of neoadjuvant chemotherapy, or if the ICIs exhibited additional effects on the immune microenvironment. More evidence and data from large-scale clinic trials are needed to explore the mechanism by which combination therapy alters the tumor microenvironment. ICIs may have great potential in cancer treatment, and examining the role of ICIs in the preoperative setting and analyzing their effect on the immune landscape may provide new insights into managing tumors and designing optimal treatment strategies.

In conclusion, radical surgery is still the cornerstone of treatment in resectable gastric cancer/GEJ. It is crucial to optimize preoperative treatment strategies, and a multidisciplinary team (MDT) cooperation is perhaps the best way to arrive at the most appropriate decision when standardized treatment modalities are lacking. Evaluating the application ICIs in perioperative setting and exploring different combination treatments is a crucial step towards optimizing cancer treatment strategies. For gastric cancer, a better understanding of the molecular subtype and immune microenvironment will likely offer more opportunities and benefits in clinical practice. We here report a patient with locally advanced GEJ adenocarcinoma, characterized as PDL1 negative, EBV negative, and $\mathrm{pMMR}$, who surprisingly achieved pCR after anti-PD-1 therapy plus chemotherapy in the neoadjuvant therapy setting. Our case indicates that a combination of ICIs plus chemotherapy as neoadjuvant treatment might offer a promising alternative for locally advanced gastric cancer patients. It is still unknown whether chemo-immunotherapy can improve the pathological response rate and OS, and this needs to be confirmed by future phase II/III clinical studies and the results of ongoing trials that are evaluating the role of neoadjuvant chemo-immunotherapy and radiotherapy in personalized perioperative treatment.

\section{Acknowledgments}

Funding: This work was supported by the Sichuan Science and Technology Program (No. 2020JDRC0025 and 2018SZ0188).

\section{Footnote}

Reporting Checklist: The authors have completed the CARE reporting checklist. Available at http://dx.doi.org/10.21037/ atm-21-434

Conflicts of Interest: All authors have completed the ICMJE uniform disclosure form (available at http://dx.doi. org/10.21037/atm-21-434). All authors report grants from Sichuan Science and Technology Program (No. 2020JDRC0025 and 2018SZ0188), during the conduct of the study. The authors have no other conflicts of interest to declare.

Ethical Statement: The authors are accountable for all aspects of the work in ensuring that questions related to the accuracy or integrity of any part of the work are appropriately investigated and resolved. All procedures performed in studies involving human participants were in accordance with the ethical standards of the institutional and/or national research committee(s) and with the Helsinki Declaration (as revised in 2013). Written informed consent was obtained from the patient for publication of this case report and any accompanying images.

Open Access Statement: This is an Open Access article distributed in accordance with the Creative Commons Attribution-NonCommercial-NoDerivs 4.0 International License (CC BY-NC-ND 4.0), which permits the noncommercial replication and distribution of the article with the strict proviso that no changes or edits are made and the 


\section{Page 10 of 13}

original work is properly cited (including links to both the formal publication through the relevant DOI and the license). See: https://creativecommons.org/licenses/by-nc-nd/4.0/.

\section{References}

1. Bray F, Ferlay J, Soerjomataram I, et al. Global cancer statistics 2018: GLOBOCAN estimates of incidence and mortality worldwide for 36 cancers in 185 countries. CA Cancer J Clin 2018;68:394-424.

2. Zeng $\mathrm{H}$, Zheng $\mathrm{R}$, Guo $\mathrm{Y}$, et al. Cancer survival in China, 2003-2005: a population-based study. Int J Cancer 2015;136:1921-30.

3. Arnold M, Rutherford MJ, Bardot A, et al. Progress in cancer survival, mortality, and incidence in seven highincome countries 1995-2014 (ICBP SURVMARK-2): a population-based study. Lancet Oncol 2019;20:1493-505.

4. Allemani C, Matsuda T, Di Carlo V, et al. Global surveillance of trends in cancer survival 2000-14 (CONCORD-3): analysis of individual records for 37513025 patients diagnosed with one of 18 cancers from 322 population-based registries in 71 countries. Lancet 2018;391:1023-75.

5. Beeharry MK, Ni ZT, Yang ZY, et al. Study protocol of a multicenter phase III randomized controlled trial investigating the efficiency of the combination of neoadjuvant chemotherapy (NAC) and neoadjuvant laparoscopic intraperitoneal hyperthermic chemotherapy (NLHIPEC) followed by R0 gastrectomy with intraoperative HIPEC for advanced gastric cancer (AGC): dragon II trial. BMC Cancer 2020;20:224.

6. Cui HB, Ge HE, Bai XY, et al. Effect of neoadjuvant chemotherapy combined with hyperthermic intraperitoneal perfusion chemotherapy on advanced gastric cancer. Exp Ther Med 2014;7:1083-8.

7. Zhandossov O, Kaussova G, Koten A. Combined treatment for gastric cancer: Immunological approach. Turk J Gastroenterol 2018;29:151-6.

8. Zheng Y, Yang X, Yan C, et al. Effect of apatinib plus neoadjuvant chemotherapy followed by resection on pathologic response in patients with locally advanced gastric adenocarcinoma: A single-arm, open-label, phase II trial. Eur J Cancer 2020;130:12-9.

9. Stahl M, Maderer A, Lordick F, et al. Perioperative chemotherapy with or without epidermal growth factor receptor blockade in unselected patients with locally advanced oesophagogastric adenocarcinoma: Randomized phase II study with advanced biomarker program of the German Cancer Society (AIO/CAO STO-0801). Eur J

\section{Li et al. Neoadjuvant immuno-chemotherapy for gastric cancer}

Cancer 2018;93:119-26.

10. Hurvitz SA, Martin M, Symmans WF, et al. Neoadjuvant trastuzumab, pertuzumab, and chemotherapy versus trastuzumab emtansine plus pertuzumab in patients with HER2-positive breast cancer (KRISTINE): a randomised, open-label, multicentre, phase 3 trial. Lancet Oncol 2018;19:115-26.

11. Topalian SL, Hodi FS, Brahmer JR, et al. Safety, activity, and immune correlates of anti-PD-1 antibody in cancer. $\mathrm{N}$ Engl J Med 2012;366:2443-54.

12. Jardim D, Goodman A, de Melo Gagliato D, et al. The Challenges of Tumor Mutational Burden as an Immunotherapy Biomarker. 2021;39:154-73.

13. Samstein RM, Lee CH, Shoushtari AN, et al. Tumor mutational load predicts survival after immunotherapy across multiple cancer types. Nat Genet 2019;51:202-6.

14. Le DT, Uram JN, Wang H, et al. PD-1 Blockade in Tumors with Mismatch-Repair Deficiency. N Engl J Med 2015;372:2509-20.

15. Le DT, Durham JN, Smith KN, et al. Mismatch repair deficiency predicts response of solid tumors to PD-1 blockade. Science 2017;357:409-13.

16. Fuchs CS, Doi T, Jang RW, et al. Safety and Efficacy of Pembrolizumab Monotherapy in Patients With Previously Treated Advanced Gastric and Gastroesophageal Junction Cancer: Phase 2 Clinical KEYNOTE-059 Trial. JAMA Oncol 2018;4:e180013.

17. Boku N, Ryu MH, Kato K, et al. Safety and efficacy of nivolumab in combination with S-1/capecitabine plus oxaliplatin in patients with previously untreated, unresectable, advanced, or recurrent gastric/ gastroesophageal junction cancer: interim results of a randomized, phase II trial (ATTRACTION-4). Ann Oncol 2019;30:250-8.

18. Bang YJ, Kang YK, Catenacci DV, et al. Pembrolizumab alone or in combination with chemotherapy as firstline therapy for patients with advanced gastric or gastroesophageal junction adenocarcinoma: results from the phase II nonrandomized KEYNOTE-059 study. Gastric Cancer 2019;22:828-37.

19. Huang J, Mo H, Zhang W, et al. Promising efficacy of SHR-1210, a novel anti-programmed cell death 1 antibody, in patients with advanced gastric and gastroesophageal junction cancer in China. Cancer 2019;125:742-9.

20. Ronellenfitsch U, Schwarzbach M, Hofheinz R, et al. Preoperative chemo(radio)therapy versus primary surgery for gastroesophageal adenocarcinoma: systematic review with meta-analysis combining individual patient and 
aggregate data. Eur J Cancer 2013;49:3149-58.

21. Cunningham D, Allum W, Stenning S, et al. Perioperative chemotherapy versus surgery alone for resectable gastroesophageal cancer. N Engl J Med 2006;355:11-20.

22. Ychou M, Boige V, Pignon J, et al. Perioperative chemotherapy compared with surgery alone for resectable gastroesophageal adenocarcinoma: an FNCLCC and FFCD multicenter phase III trial. J Clin Oncol 2011;29:1715-21.

23. Nakamura Y, Yamanaka T, Chin K, et al. Survival Outcomes of Two Phase 2 Studies of Adjuvant Chemotherapy with S-1 Plus Oxaliplatin or Capecitabine Plus Oxaliplatin for Patients with Gastric Cancer After D2 Gastrectomy. Ann Surg Oncol 2019;26:465-72.

24. Shitara K, Chin K, Yoshikawa T, et al. Phase II study of adjuvant chemotherapy of S-1 plus oxaliplatin for patients with stage III gastric cancer after D2 gastrectomy. Gastric Cancer 2017;20:175-81.

25. Fuse N, Bando H, Chin K, et al. Adjuvant capecitabine plus oxaliplatin after D2 gastrectomy in Japanese patients with gastric cancer: a phase II study. Gastric Cancer 2017;20:332-40.

26. Al-Batran SE, Homann N, Pauligk C, et al. Perioperative chemotherapy with fluorouracil plus leucovorin, oxaliplatin, and docetaxel versus fluorouracil or capecitabine plus cisplatin and epirubicin for locally advanced, resectable gastric or gastro-oesophageal junction adenocarcinoma (FLOT4): a randomised, phase 2/3 trial. Lancet 2019;393:1948-57.

27. Smyth EC, Verheij M, Allum W, et al. Gastric cancer: ESMO Clinical Practice Guidelines for diagnosis, treatment and follow-up. Ann Oncol 2016;27:v38-v49.

28. Wang Z, Yan J, Hu W, et al. Adjuvant chemotherapy provided survival benefit for stage T2N0 gastric cancer with high-risk factors. Neoplasma 2018;65:592-8.

29. Sun KK, Wang QH, Wu YY. Challenges surrounding postoperative adjuvant chemotherapy for T2N0 gastric cancer. Oncol Lett 2020;20:126.

30. van Hagen P, Hulshof MC, van Lanschot JJ, et al. Preoperative chemoradiotherapy for esophageal or junctional cancer. N Engl J Med 2012;366:2074-84.

31. Tepper J, Krasna MJ, Niedzwiecki D, et al. Phase III trial of trimodality therapy with cisplatin, fluorouracil, radiotherapy, and surgery compared with surgery alone for esophageal cancer: CALGB 9781. J Clin Oncol 2008;26:1086-92.

32. Macdonald JS, Smalley SR, Benedetti J, et al. Chemoradiotherapy after surgery compared with surgery alone for adenocarcinoma of the stomach or gastroesophageal junction. N Engl J Med 2001;345:725-30.

33. Stiekema J, Trip AK, Jansen EP, et al. Does adjuvant chemoradiotherapy improve the prognosis of gastric cancer after an $\mathrm{r} 1$ resection? Results from a dutch cohort study. Ann Surg Oncol 2015;22:581-8.

34. Slagter AE, Jansen E, van Laarhoven H, et al. CRITICS-II: a multicentre randomised phase II trial of neo-adjuvant chemotherapy followed by surgery versus neo-adjuvant chemotherapy and subsequent chemoradiotherapy followed by surgery versus neoadjuvant chemoradiotherapy followed by surgery in resectable gastric cancer. BMC Cancer 2018;18:877.

35. Cats A, Jansen E, van Grieken N, et al. Chemotherapy versus chemoradiotherapy after surgery and preoperative chemotherapy for resectable gastric cancer (CRITICS): an international, open-label, randomised phase 3 trial. Lancet Oncol 2018;19:616-28.

36. Cohen R, Pudlarz T, Garcia-Larnicol M, et al. Localized MSI/dMMR gastric cancer patients, perioperative immunotherapy instead of chemotherapy: The GERCOR NEONIPIGA phase II study is opened to recruitment. Bull Cancer 2020;107:438-46.

37. Smyth E, Knödler M, Giraut A, et al. VESTIGE: Adjuvant Immunotherapy in Patients With Resected Esophageal, Gastroesophageal Junction and Gastric Cancer Following Preoperative Chemotherapy With High Risk for Recurrence (N+ and/or R1): An Open Label Randomized Controlled Phase-2-Study. Front Oncol 2020;9:1320.

38. Bang YJ, Van Cutsem E, Fuchs CS, et al. KEYNOTE-585: Phase III study of perioperative chemotherapy with or without pembrolizumab for gastric cancer. Future Oncol 2019;15:943-52.

39. Weber J, Mandala M, Del Vecchio M, et al. Adjuvant Nivolumab versus Ipilimumab in Resected Stage III or IV Melanoma. N Engl J Med 2017;377:1824-35.

40. Eggermont AM, Chiarion-Sileni V, Grob JJ, et al. Prolonged Survival in Stage III Melanoma with Ipilimumab Adjuvant Therapy. N Engl J Med 2016;375:1845-55.

41. Eggermont AM, Chiarion-Sileni V, Grob JJ, et al. Adjuvant ipilimumab versus placebo after complete resection of high-risk stage III melanoma (EORTC 18071): a randomised, double-blind, phase 3 trial. Lancet Oncol 2015;16:522-30.

42. Zimmer L, Livingstone E, Hassel JC, et al. Adjuvant nivolumab plus ipilimumab or nivolumab monotherapy versus placebo in patients with resected stage IV melanoma 
with no evidence of disease (IMMUNED): a randomised, double-blind, placebo-controlled, phase 2 trial. Lancet 2020;395:1558-68.

43. Muro K, Chung H, Shankaran V, et al. Pembrolizumab for patients with PD-L1-positive advanced gastric cancer (KEYNOTE-012): a multicentre, open-label, phase 1b trial. Lancet Oncol 2016;17:717-26.

44. Kang YK, Boku N, Satoh T, et al. Nivolumab in patients with advanced gastric or gastro-oesophageal junction cancer refractory to, or intolerant of, at least two previous chemotherapy regimens (ONO-4538-12, ATTRACTION-2): a randomised, double-blind, placebocontrolled, phase 3 trial. Lancet 2017;390:2461-71.

45. Shitara K, Özgüroğlu M, Bang Y, et al. Pembrolizumab versus paclitaxel for previously treated, advanced gastric or gastro-oesophageal junction cancer (KEYNOTE-061): a randomised, open-label, controlled, phase 3 trial. Lancet 2018;392:123-33.

46. Shitara K, Van Cutsem E, Bang Y, et al. Efficacy and Safety of Pembrolizumab or Pembrolizumab Plus Chemotherapy vs Chemotherapy Alone for Patients With First-line, Advanced Gastric Cancer: The KEYNOTE-062 Phase 3 Randomized Clinical Trial. JAMA Oncol 2020;6:1571-80.

47. Janjigian YY, Bendell J, Calvo E, et al. CheckMate-032 Study: Efficacy and Safety of Nivolumab and Nivolumab Plus Ipilimumab in Patients With Metastatic Esophagogastric Cancer. J Clin Oncol 2018;36:2836-44.

48. Janjigian YY, Maron SB, Chatila WK, et al. First-line pembrolizumab and trastuzumab in HER2-positive oesophageal, gastric, or gastro-oesophageal junction cancer: an open-label, single-arm, phase 2 trial. Lancet Oncol 2020;21:821-31.

49. Chung HC, Bang YJ, C SF, et al. First-line pembrolizumab/placebo plus trastuzumab and chemotherapy in HER2-positive advanced gastric cancer: KEYNOTE-811. Future Oncol 2021;17:491-501.

50. Herbst RS, Arkenau H, Santana-Davila R, et al. Ramucirumab plus pembrolizumab in patients with previously treated advanced non-small-cell lung cancer, gastro-oesophageal cancer, or urothelial carcinomas (JVDF): a multicohort, non-randomised, open-label, phase 1a/b trial. Lancet Oncol 2019;20:1109-23.

51. Derer A, Spiljar M, Bäumler M, et al. Chemoradiation Increases PD-L1 Expression in Certain Melanoma and Glioblastoma Cells. Front Immunol 2016;7:610.

52. Merhi M, Raza A, Inchakalody V, et al. Persistent antiNY-ESO-1-specific T cells and expression of differential biomarkers in a patient with metastatic gastric cancer benefiting from combined radioimmunotherapy treatment: a case report. J Immunother Cancer 2020;8:e01278.

53. Thuss-Patience PC, Hofheinz RD, Arnold D, et al. Perioperative chemotherapy with docetaxel, cisplatin and capecitabine (DCX) in gastrooesophageal adenocarcinoma: a phase II study of the Arbeitsgemeinschaft Internistische Onkologie (AIO) \{dagger\}. Ann Oncol 2012;23:2827-34.

54. Schulz C, Kullmann F, Kunzmann V, et al. NeoFLOT: Multicenter phase II study of perioperative chemotherapy in resectable adenocarcinoma of the gastroesophageal junction or gastric adenocarcinoma-Very good response predominantly in patients with intestinal type tumors. Int J Cancer 2015;137:678-85.

55. Al-Batran SE, Hofheinz RD, Pauligk C, et al.

Histopathological regression after neoadjuvant docetaxel, oxaliplatin, fluorouracil, and leucovorin versus epirubicin, cisplatin, and fluorouracil or capecitabine in patients with resectable gastric or gastro-oesophageal junction adenocarcinoma (FLOT4-AIO): results from the phase 2 part of a multicentre, open-label, randomised phase 2/3 trial. Lancet Oncol 2016;17:1697-708.

56. Wan T, Zhang XF, Liang C, et al. The Prognostic Value of a Pathologic Complete Response After Neoadjuvant Therapy for Digestive Cancer: Systematic Review and Meta-Analysis of 21 Studies. Ann Surg Oncol 2019;26:1412-20.

57. Cancer Genome Atlas Research Network. Comprehensive molecular characterization of gastric adenocarcinoma. Nature 2014;513:202-9.

58. Kim ST, Cristescu R, Bass AJ, et al. Comprehensive molecular characterization of clinical responses to PD-1 inhibition in metastatic gastric cancer. Nat Med 2018;24:1449-58.

59. Shevtsov M, Sato H, Multhoff G, et al. Novel Approaches to Improve the Efficacy of Immuno-Radiotherapy. Front Oncol 2019;9:156.

60. Zitvogel L, Kepp O, Senovilla L, et al. Immunogenic tumor cell death for optimal anticancer therapy: the calreticulin exposure pathway. Clin Cancer Res 2010;16:3100-4.

61. Hodge JW, Garnett CT, Farsaci B, et al. Chemotherapyinduced immunogenic modulation of tumor cells enhances killing by cytotoxic $\mathrm{T}$ lymphocytes and is distinct from immunogenic cell death. Int J Cancer 2013;133:624-36.

62. Li JY, Chen YP, Li YQ, et al. Chemotherapeutic and targeted agents can modulate the tumor microenvironment and increase the efficacy of immune checkpoint blockades. 
Mol Cancer 2021;20:27.

63. Blumenthal GM, Zhang L, Zhang H, et al. Milestone Analyses of Immune Checkpoint Inhibitors, Targeted Therapy, and Conventional Therapy in Metastatic NonSmall Cell Lung Cancer Trials: A Meta-analysis. JAMA Oncol 2017;3:e171029.

64. Salem ME, Puccini A, Grothey A, et al. Landscape of Tumor Mutation Load, Mismatch Repair Deficiency, and PD-L1 Expression in a Large Patient Cohort of Gastrointestinal Cancers. Mol Cancer Res 2018;16:805-12.

65. Zhao X, Ren Y, Hu Y, et al. Neoadjuvant chemotherapy versus neoadjuvant chemoradiotherapy for cancer of the esophagus or the gastroesophageal junction: A meta-analysis based on clinical trials. PLoS One 2018;13:e0202185.

66. Denkert C, von Minckwitz G, Darb-Esfahani S, et al. Tumour-infiltrating lymphocytes and prognosis in different subtypes of breast cancer: a pooled analysis of 3771 patients treated with neoadjuvant therapy. Lancet Oncol 2018;19:40-50.

67. Gide TN, Quek C, Menzies AM, et al. Distinct Immune Cell Populations Define Response to Anti-PD-1 Monotherapy and Anti-PD-1/Anti-CTLA-4 Combined Therapy. Cancer Cell 2019;35:238-255.e6.

68. Sasaki A, Nakamura Y, Mishima S, et al. Predictive factors for hyperprogressive disease during nivolumab as antiPD1 treatment in patients with advanced gastric cancer. Gastric Cancer 2019;22:793-802.

69. Coffelt SB, Wellenstein MD, de Visser KE. Neutrophils in cancer: neutral no more. Nat Rev Cancer 2016;16:431-46.

70. Uribe-Querol E, Rosales C. Neutrophils in Cancer: Two Sides of the Same Coin. J Immunol Res 2015;2015:983698.

71. Di Carlo E, Forni G, Lollini P, et al. The intriguing role of polymorphonuclear neutrophils in antitumor reactions. Blood 2001;97:339-45.

72. Guerriero JL. Macrophages: The Road Less Traveled, Changing Anticancer Therapy. Trends Mol Med

Cite this article as: Li X, Huang Q, Lei Y, Zheng X, Dai S, Leng W, Liu M. Locally advanced gastroesophageal junction cancer with pathological complete response to neoadjuvant therapy: a case report and literature review. Ann Transl Med 2021;9(6):513. doi: 10.21037/atm-21-434
2018;24:472-89.

73. Ruffell B, Affara NI, Coussens LM. Differential macrophage programming in the tumor microenvironment. Trends Immunol 2012;33:119-26.

74. Tumeh PC, Harview CL, Yearley JH, et al. PD-1 blockade induces responses by inhibiting adaptive immune resistance. Nature 2014;515:568-71.

75. Derks S, de Klerk LK, Xu X, et al. Characterizing diversity in the tumor-immune microenvironment of distinct subclasses of gastroesophageal adenocarcinomas. Ann Oncol 2020;31:1011-20.

76. Chen DS, Mellman I. Elements of cancer immunity and the cancer-immune set point. Nature 2017;541:321-30.

77. Kim TS, da Silva E, Coit DG, et al. Intratumoral Immune Response to Gastric Cancer Varies by Molecular and Histologic Subtype. Am J Surg Pathol 2019;43:851-60.

78. Fridman WH, Zitvogel L, Sautès-Fridman C, et al. The immune contexture in cancer prognosis and treatment. Nat Rev Clin Oncol 2017;14:717-34.

79. Yu Y, Ma X, Zhang Y, et al. Changes in Expression of Multiple Checkpoint Molecules and Infiltration of Tumor Immune Cells after Neoadjuvant Chemotherapy in Gastric Cancer. J Cancer 2019;10:2754-63.

80. Mesnage SJL, Auguste A, Genestie C, et al. Neoadjuvant chemotherapy (NACT) increases immune infiltration and programmed death-ligand 1 (PD-L1) expression in epithelial ovarian cancer (EOC). Ann Oncol 2017;28:651-7.

81. Wakatsuki K, Sho M, Yamato I, et al. Clinical impact of tumor-infiltrating CD45 $\mathrm{RO}^{+}$memory T cells on human gastric cancer. Oncol Rep 2013;29:1756-62.

82. Zhang N, Cao M, Duan Y, et al. Prognostic role of tumor-infiltrating lymphocytes in gastric cancer: a metaanalysis and experimental validation. Arch Med Sci 2019;16:1092-103.

(English Language Editor: J. Gray) 